\title{
Obesity Fact Sheet in Korea, 2020: Prevalence of Obesity by Obesity Class from 2009 to 2018
}

Ga Eun Nam', Yang-Hyun Kim¹, Kyungdo Han², Jin-Hyung Jung ${ }^{3}$, Eun-Jung Rhee, Won-Young Lee, ${ }^{4, *}$ On Behalf of the Taskforce Team of the Obesity Fact Sheet of the Korean Society for the Study of Obesity

'Department of Family Medicine, Korea University College of Medicine, Seoul;: 2Department of Statistics and Actuarial Science, Soongsil University, Seoul; ${ }^{3}$ Department of Biostatistics, College of Medicine, The Catholic University of Korea, Seoul; ${ }^{4}$ Division of Endocrinology and Metabolism, Department of Internal Medicine, Kangbuk Samsung Hospital, Sungkyunkwan University School of Medicine, Seoul, Korea

Background: We examined the prevalence of different obesity classes in South Korea based on the 2020 Obesity Fact Sheet.

Methods: Individuals $\geq 20$ years who underwent a health examination provided by the Korean National Health Insurance System between 2009 and 2018 were included and the prevalence of class I, II, and III obesity was calculated for the total sample and age, sex, and region subgroups.

Results: From 2009 to 2018, the prevalence of all obesity classes increased across all sex and age groups and all regions. In the study population as a whole, the prevalence of class I, II, and III obesity was $29.1 \%, 3.2 \%$, and $0.3 \%$ in 2009 and $32.5 \%, 5.2 \%$, and $0.81 \%$ in 2018, respectively. Among young-aged individuals, the prevalence of each obesity class was $23.7 \%, 3.6 \%$, and $0.44 \%$ in 2009 and $28.3 \%, 6.9 \%$, and $1.61 \%$ in 2018 , respectively. The prevalence among middle-aged individuals was $31.6 \%, 3.1 \%$, and $0.24 \%$ in 2009 and $33.6 \%, 4.8 \%$, and $0.59 \%$ in 2018; and among elderly individuals was 31.9\%, 3.1\%, and $0.21 \%$ in 2009 and 35.5\%, 3.9\%, and $0.32 \%$ in 2018 . The increase in the prevalence of all obesity classes among young adults was dramatic. In particular, the class III obesity prevalence increased up to 3.8- and 3.5-fold between 2009 and 2018 in young men and women. Conclusion: Based on the 2020 Obesity Fact Sheet, there was a dramatic increase in the prevalence of class II and III obesity from 2009 to 2018 among young adults, as well as the population as a whole. Optimal strategies for the prevention and treatment of obesity are needed considering the recent obesity epidemic in South Korea.
Received May 14, 2021

Reviewed June 12, 2021

Accepted June 13, 2021

*Corresponding author Won-Young Lee

https://orcid.org/0000-0002-1082-7592

Division of Endocrinology and Metabolism, Department of Internal Medicine, Kangbuk Samsung Hospital, Sungkyunkwan University School of Medicine, 29 Saemunan-ro, Jongno-gu, Seoul 03181, Korea

Tel: +82-2-2001-2579

Fax: +82-2-2001-1588

E-mail: drlwy@hanmail.net

Key words: Obesity, Obesity class, Prevalence, Fact sheet, National Health Insurance System

\section{INTRODUCTION}

Obesity is a complex, multifactorial chronic disease that is associated with higher rates of comorbidities such as hypertension, type 2 diabetes mellitus, dyslipidemia, obstructive sleep apnea, steatohepatitis, cardiovascular diseases, and several types of cancer, as well as higher risk of death from such comorbidities. ${ }^{1,2}$ Obesity substantially affects patient quality of life, limits economic and social activity opportunities, and imposes a considerable financial burden on patients and society. Notwithstanding the many efforts to reduce the obesity epidemic and its impact, obesity remains a major public health issue worldwide. ${ }^{1,2}$ According to the 2019 Obesity Fact Sheet by the Korean Society for the Study of Obesity (KSSO), the prevalence of obesity and abdominal obesity in Korea continuously increased in the adult population and among both sexes and nearly all age groups between 2009 and 2018. ${ }^{3}$ The rapid increase in obesity prevalence is a global phenomenon and a major healthcare challenge across both developed and developing countries. Moreover, a steep increment of the prevalence of morbid obesity warrants further research and the development of a variety 
of management tools. ${ }^{4}$

KSSO has invested considerable effort into investigating the epidemiology of obesity and obesity-related comorbidities in order to improve obesity and obesity-related health outcomes across Korea. For that reason, the KSSO has published Obesity Fact Sheets for Korea since 2015 in collaboration with the Korean National Health Insurance Corporation (NHIC). The Obesity Fact Sheets sought to evaluate the status of obesity and obesity-related diseases and impacts of obesity, as well as to provide health statistics for the development of national health policies on obesity in Korea. The 2020 Obesity Fact Sheet described recent trends in the prevalence of obesity by obesity class in age and sex groups, and region of South Korea. Hence, in this study, we reported its rationale and methods and discussed the prevalence of obesity classes in South Korea based on the 2020 Obesity Fact Sheet.

\section{METHODS}

\section{Data source and study population}

This study was based on the national health checkup dataset offered by the Korean National Health Insurance Service (NHIS). The Korean NHIC manages the NHIS, which is a universal and mandatory health insurance system covering $97 \%$ of Koreans and provides at least biennial health screenings for all insured Koreans. The NHIS possesses a database of nearly the whole South Korean population including demographic information, health examination data, and diagnosis of diseases and medical treatment identified by the International Classification of Diseases, 10th revision and prescription codes. We analyzed data on individuals aged $\geq 20$ years who underwent a health examination provided by the Korean NHIS between January 1, 2009, and December 31, 2018. The Institutional Review Board of the Kangbuk Samsung Hospital approved this study protocol (No. KBSMC 2020-05-002).

\section{Definition of obesity and obesity classes}

Trained staff measured participant height and body weight for anthropometric assessment. We calculated body mass index (BMI) by dividing body weight $(\mathrm{kg})$ by the square of the height $(\mathrm{m})$. We defined obesity as a BMI $\geq 25 \mathrm{~kg} / \mathrm{m}^{2}$, according to the Asia-Pacific criteria of the World Health Organization guidelines. Obesity classes were defined using the 2018 KSSO Obesity Guideline for the Management of Obesity as follows, class I obesity, BMI $25.0-29.9 \mathrm{~kg} / \mathrm{m}^{2}$; class II obesity, $30.0-34.9 \mathrm{~kg} / \mathrm{m}^{2}$; class III obesity, $\geq 35.0 \mathrm{~kg} / \mathrm{m}^{2}$.

\section{Statistical analysis}

All statistical analyses were performed using SAS version 9.4 (SAS Institute Inc., Cary, NC, USA). We presented the prevalence of obesity and obesity classes in the study population as a whole, as well as prevalence by age group (young: 20-39 years, middle-aged: 40-64 years, and elderly: $\geq 65$ years), sex, and region of South Korea.

\section{RESULTS}

\section{Prevalence of obesity by obesity class (2009-2018)}

The prevalence of overall obesity in 2009 was $32.6 \%$ and increased by 1.18 -fold to $38.5 \%$ in 2018 , respectively. Fig. 1 presents the prevalence of obesity by obesity class from 2009 to 2018 in the whole population and by sex. The prevalence of all three classes of obesity increased between 2009 and 2018 for all groups (all $P$ for trend $<0.001)$. In the whole sample, the prevalence of class I, II, and III obesity in 2009 was $29.1 \%, 3.2 \%$, and $0.3 \%$, respectively, and was increased by 1.12-, 1.63-, 2.79-fold in 2018 to $32.5 \%, 5.2 \%$, and $0.81 \%$, respectively (Fig. $1 \mathrm{~A}$ ). During this period, the prevalence of each obesity class increased among both men and women ( $P$ for trend $<0.001$ ). The prevalence of class I, II, and III obesity in men was 33.5\%, 3.4\%, and $0.26 \%$ in 2009 and 39.4\%, 6.0\%, and $0.86 \%$ in 2018 . In women, the prevalence was $23.7 \%, 3.1 \%$, and $0.35 \%$ in 2009 and $24.6 \%, 4.3 \%$, and $0.75 \%$ in 2018 , respectively (Fig. 1B, C).

\section{Prevalence of obesity by obesity class in age and sex groups (2009-2018)}

Fig. 2 shows the prevalence of each obesity class from 2009 and 2018 among different age groups. Among all age groups, the prevalence of class I, II, and III obesity increased during this period (all $P$ for trend <0.001). Among young individuals, the prevalence of class I, II, and III obesity was $23.7 \%, 3.6 \%$, and $0.44 \%$ in 2009 and $28.3 \%, 6.9 \%$, and $1.61 \%$ in 2018 , respectively. The prevalence of each obesity class among middle-aged individuals was $31.6 \%, 3.1 \%$, 
(4)

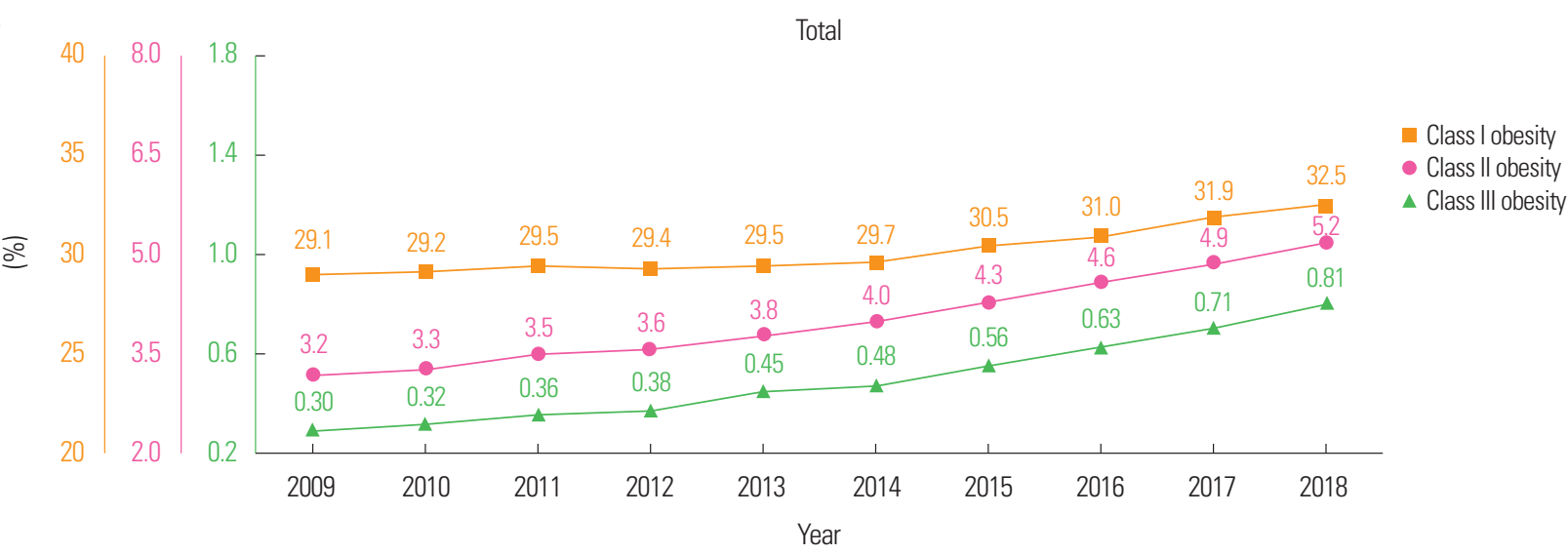

B

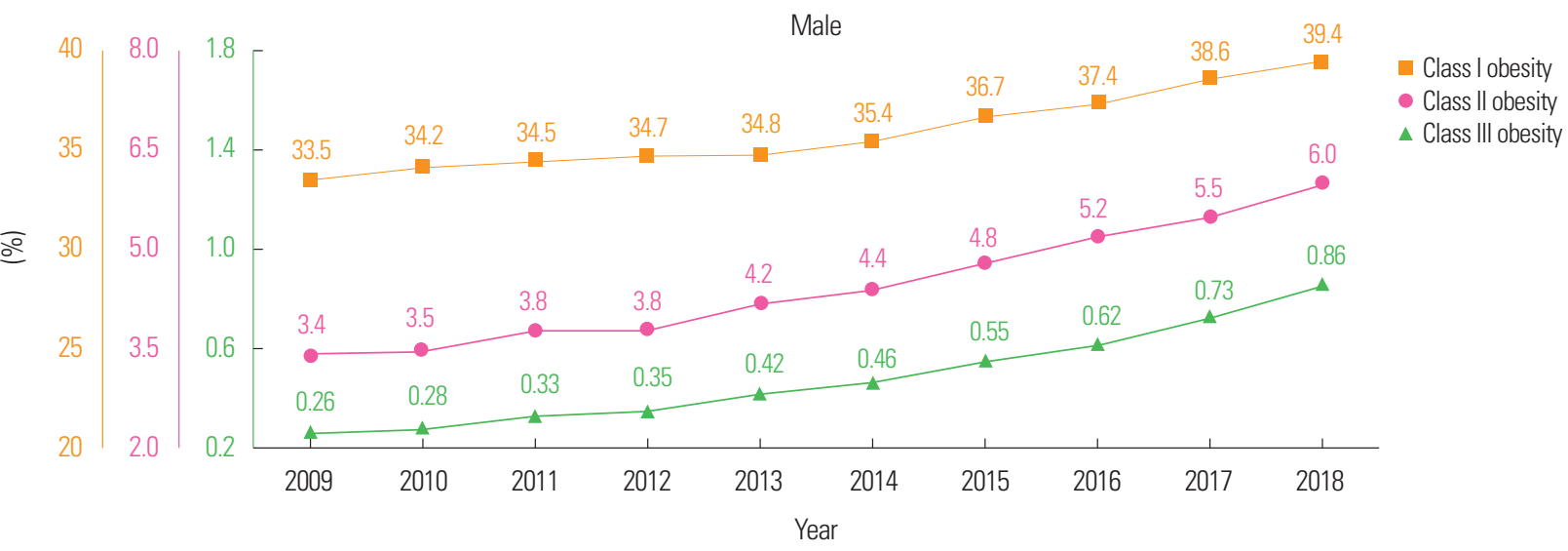

C

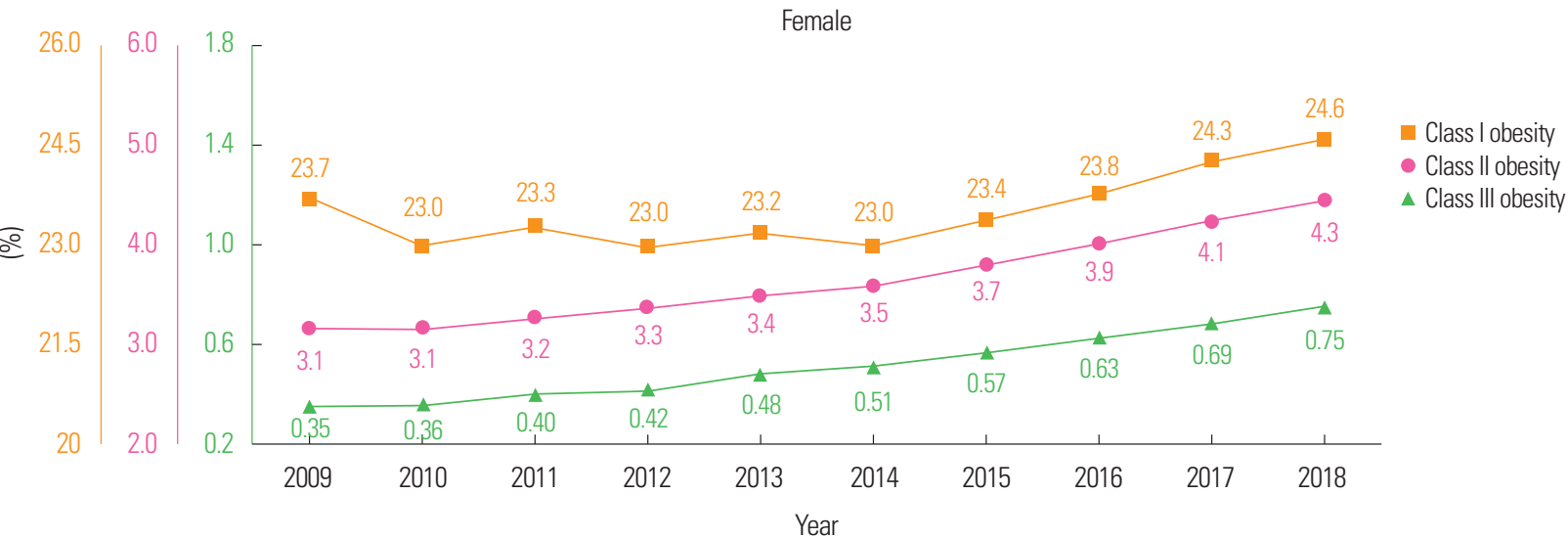

Figure 1. Prevalence of obesity by obesity class (2009-2018) in the total population (A), men (B), and women (C) (all Pfor trend $<0.001$ ). Orange, pink, and green lines represent the prevalence of class I, class II, and class III obesity, respectively.

and $0.24 \%$ in 2009 and $33.6 \%, 4.8 \%$, and $0.59 \%$ in 2018. Among elderly individuals, the prevalence was $31.9 \%, 3.1 \%$, and $0.21 \%$ in 2009 and $35.5 \%, 3.9 \%$, and $0.32 \%$ in 2018 .

The age-specific prevalence of obesity classes increased between 2009 and 2018 among both men and women, as shown in Fig. 3. In 2018 , the prevalence of class I obesity for young, middle-aged, and elderly men was $37.8 \%, 41.4 \%$, and $34.8 \%$, respectively. The prevalence of class II obesity was $8.9 \%, 5.4 \%$, and $2.6 \%$ and class III obesity was $1.85 \%, 0.53 \%$, and $0.12 \%$ in young, middle-aged, and elderly men, respectively. Compared to 2009, 3.8-fold increases in class III obesity were observed in young and middle-aged men in 2018. In 2018, the prevalence of class I obesity among young, mid- 
A

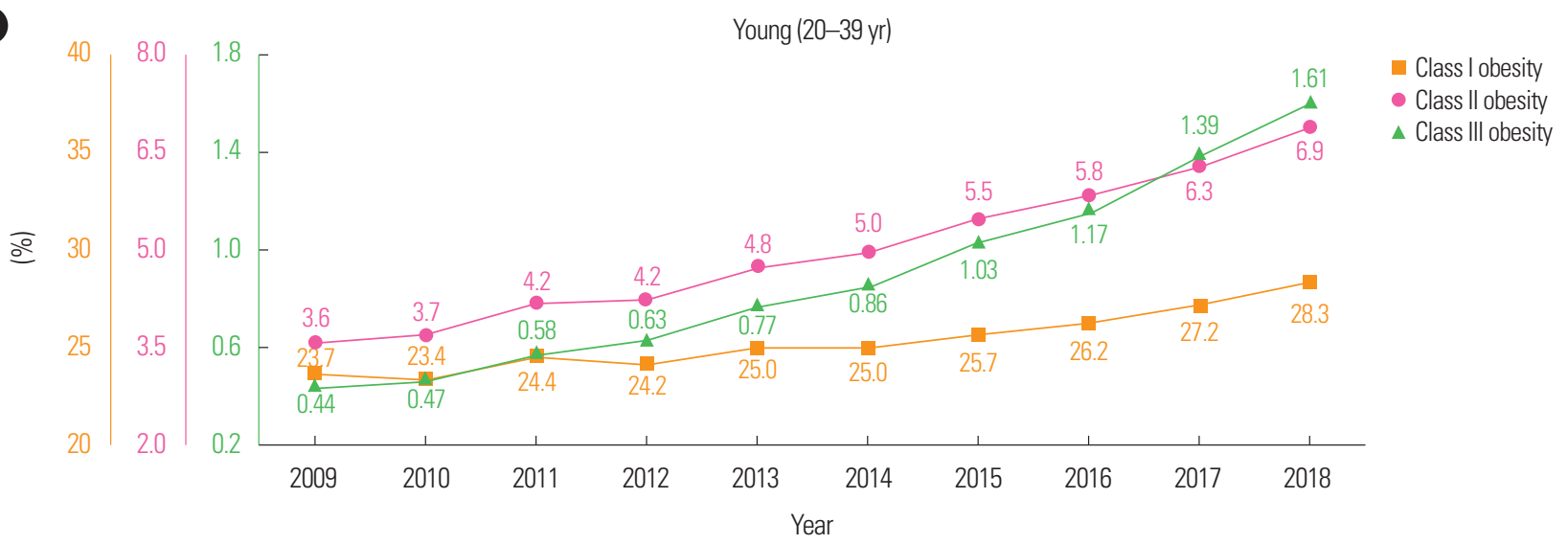

B

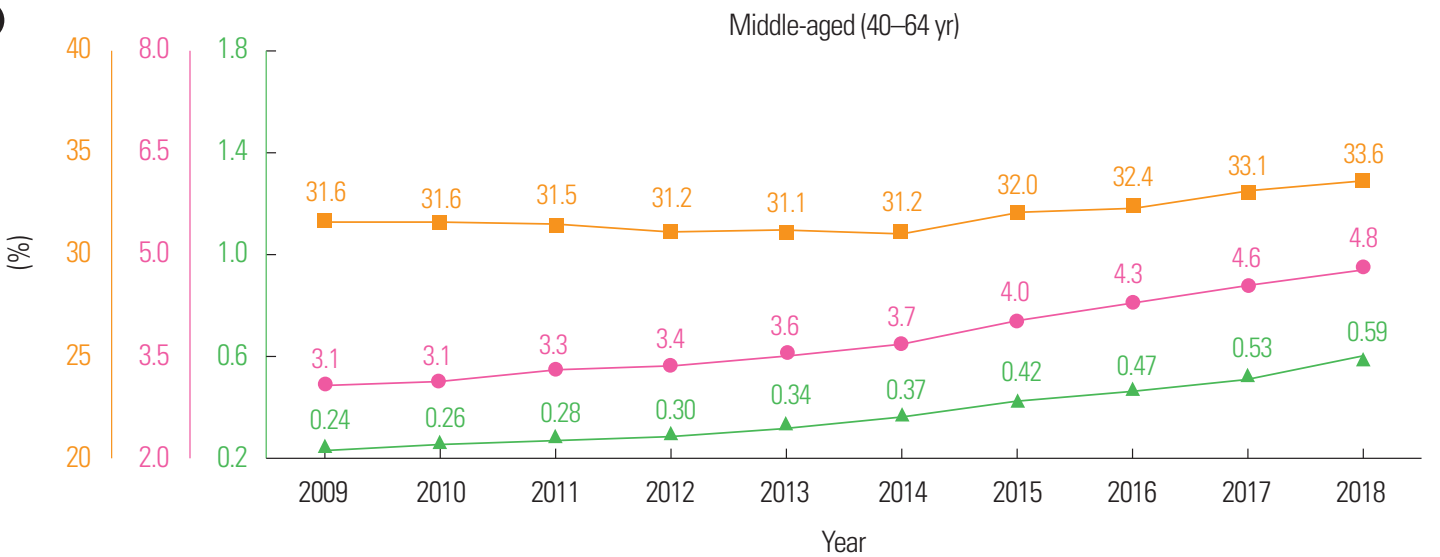

- Class I obesity

- Class II obesity

- Class III obesity

C

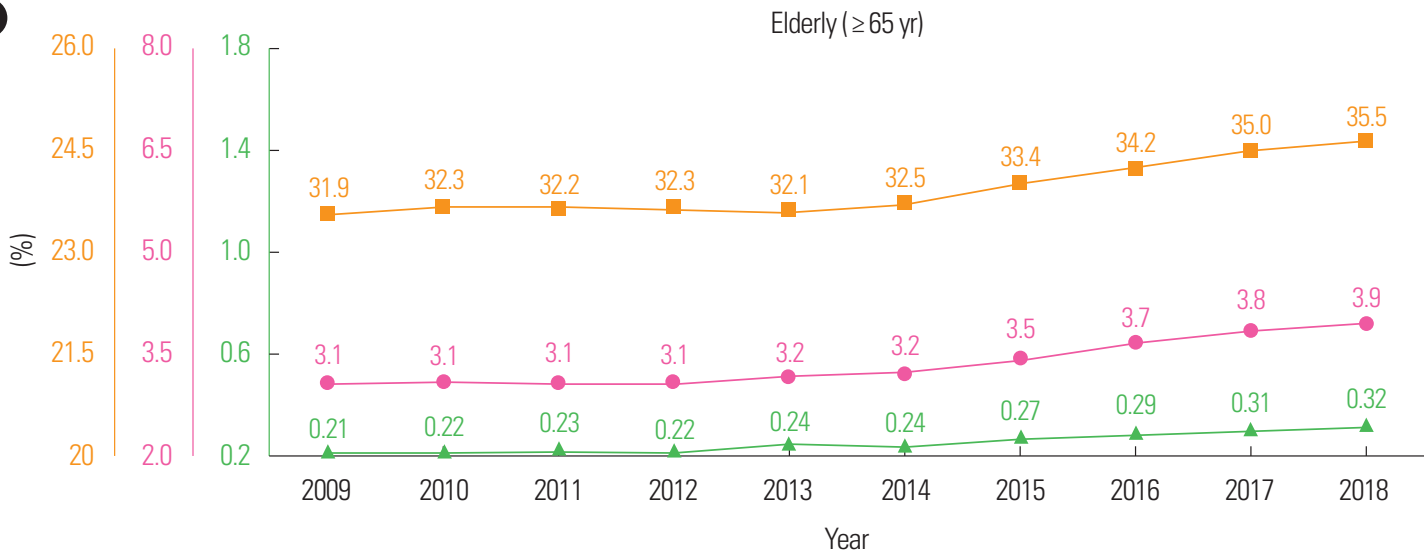

- Class I obesity

- Class II obesity

- Class III obesity

Figure 2. Prevalence of obesity classes (2009-2018) in young (A), middle-aged (B), and elderly (C) individuals (all $P$ for trend $<0.001$ ). Orange, pink, and green lines represent the prevalence of class I, class II, and class III obesity, respectively.

dle-aged, and elderly women was $12.9 \%, 25.2 \%$, and $36.1 \%$, respectively. The prevalence of class II obesity was $3.7 \%, 4.2 \%$, and $5.2 \%$ and class III obesity prevalence was $1.22 \%, 0.67 \%$, and $0.51 \%$ in young, middle-aged, and elderly women, respectively. There were 2.1- and 3.5-fold increases in class II and class III obesity in young females in 2018 compared to 2009.

\section{Prevalence of obesity classes by region}

The prevalence of overall obesity and all three classes of obesity increased in all regions of South Korea in 2018 compared to 2009 (Fig. 4). In 2018, Jeju-si had the highest prevalence of all obesity classes and the second highest prevalence of all obesity classes was observed in Gangwon-do. 

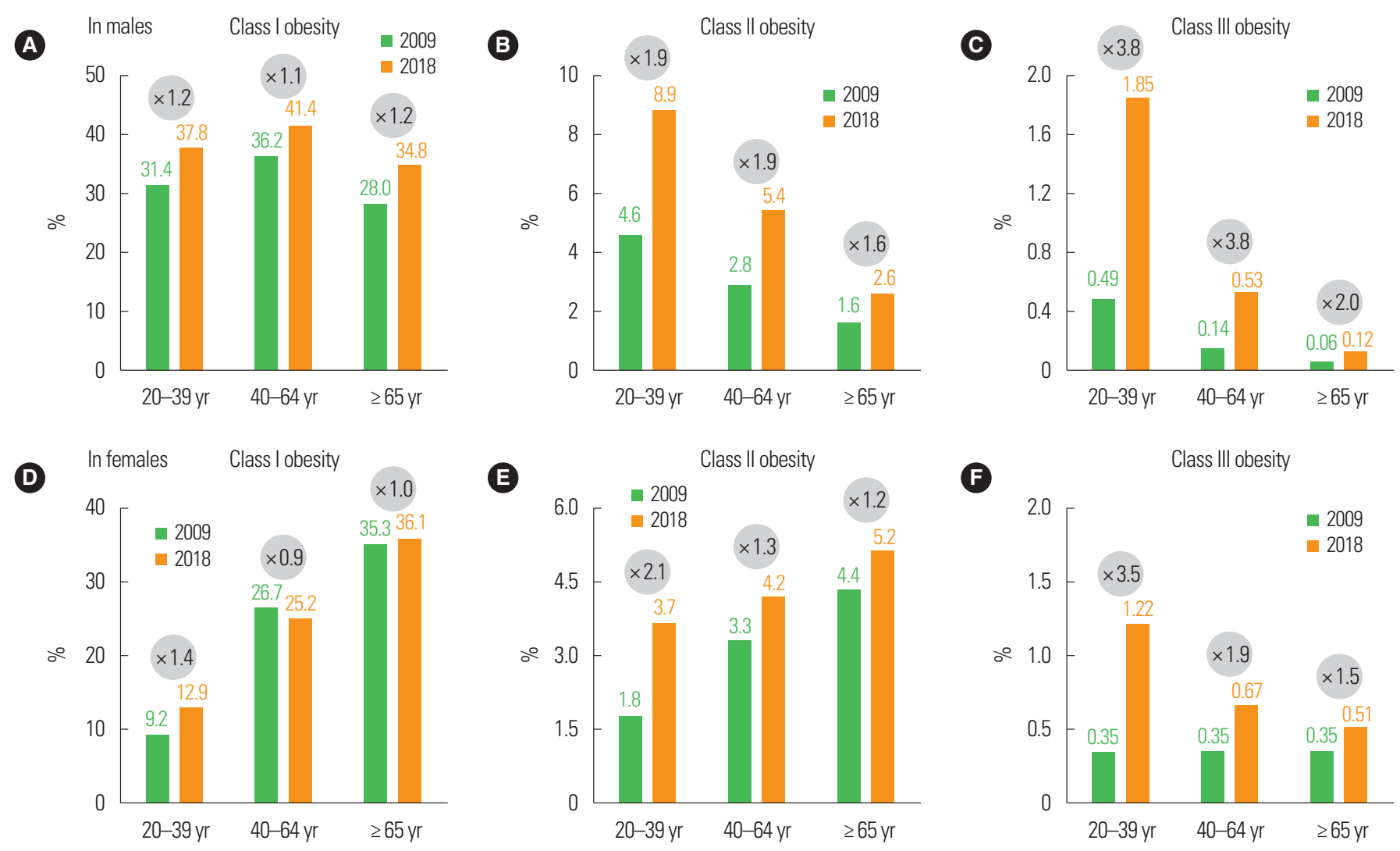

Figure 3. Age-specific prevalence of obesity classes between 2009 and 2018 in men (A-C) and women (D-F).

\section{DISCUSSION}

Based on the 2020 Obesity Fact Sheet in Korea, the prevalence of class I, II, and III obesity continued to increase from 2009 to 2018 in the population as a whole and in all subgroups. Among the three classes of obesity, the increase in the prevalence of class III obesity during this period was the greatest in the total population and in all subgroups. The increase in the prevalence of class II and III obesity was greater among men than in women, and was greater in young individuals than among middle-aged and elderly individuals. Between 2009 and 2018, the prevalence of class III obesity increased 3.8- and 3.5-fold between 2009 and 2018 in young males and females, respectively. This factsheet highlighted a steep increase in class III obesity (morbid obesity) among the population as a whole and all subgroups in South Korea. Furthermore, increases in the prevalence of all obesity classes including class II and III obesity were the most prominent in young individuals during the past decade.

Globally, there has been a faster increase in the prevalence of higher-grade obesity or morbid obesity. A report from the National Center for Health Statistics suggested that the prevalence of overall obesity in the United States increased more than 36\% from 1999 to $2014 .{ }^{6}$ In addition, an alarming prevalence increase in individual BMI categories was observed; the rate of overweight remained roughly same, while the rate of extreme obesity increased more than 6-fold to $6.6 \%$ in 2009-2010 compared to in 1962, based on National Health and Nutrition Examination Survey data. ${ }^{6,7}$ In South Korea, the prevalence of class II and III obesity increased rapidly based on the 2020 Obesity Fact Sheet. Obesity is related to higher rates of comorbidities such as hypertension, dyslipidemia, type 2 diabetes mellitus, obstructive sleep apnea, certain cancers, fatty liver, gastroesophageal reflux, arthritis, and polycystic ovary syndrome. ${ }^{8,9}$ Studies revealed evidently that the risk of these diseases rises in morbid obesity. One study reported that the risk of developing type 2 diabetes mellitus surged at a BMI $\geq 27.2 \mathrm{~kg} / \mathrm{m}^{2}{ }^{10}$ Obesity is associated with higher rates of mortality caused by obesity-related comorbidities at higher BMI levels. A study reported 

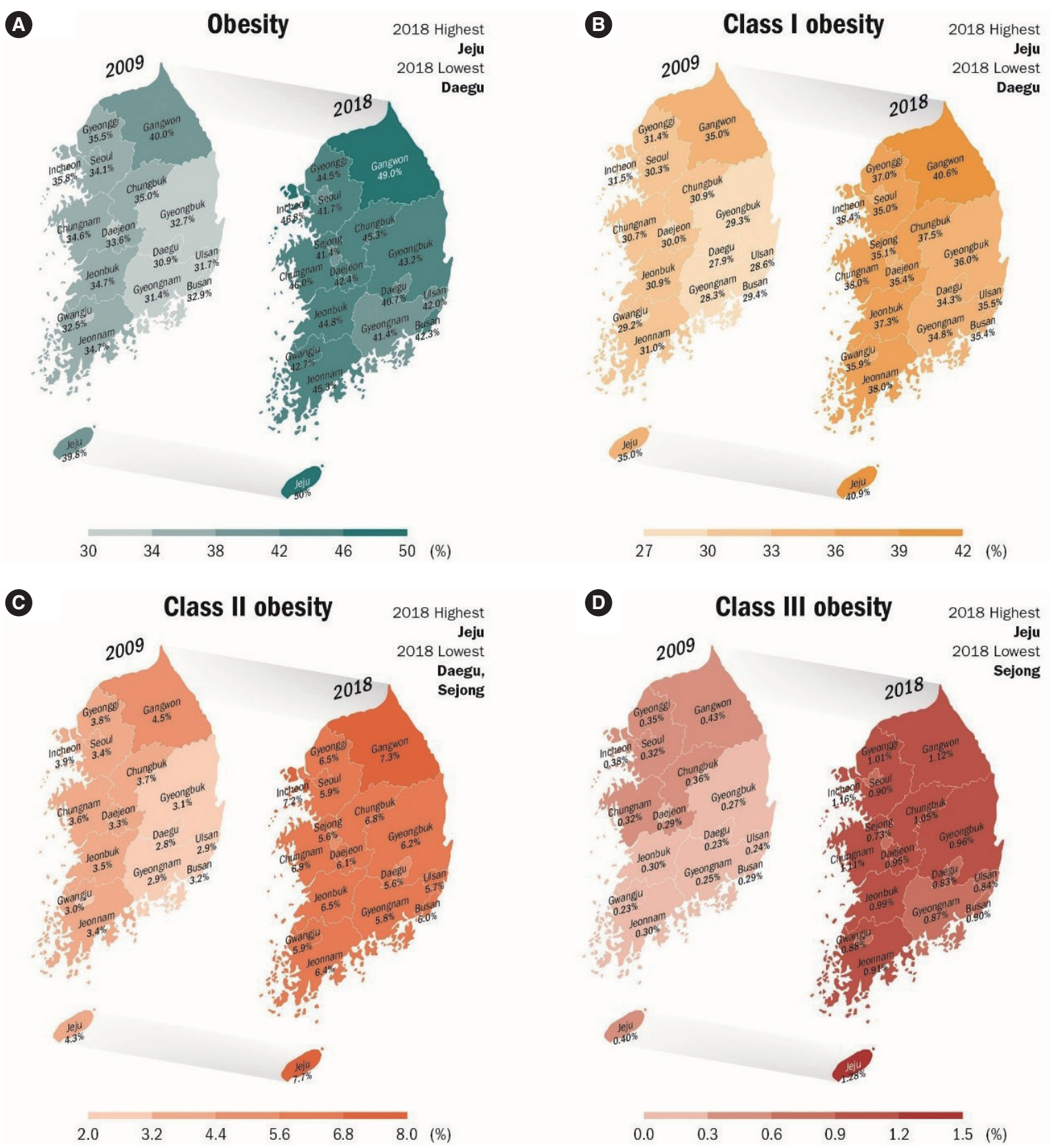

Figure 4. Prevalence of overall (A), class I (B), II (C), and III (D) obesity between 2009 and 2018 by region of South Korea.

that both class II and III obesity were associated with increased risk of all-cause mortality while class I obesity was not. ${ }^{11}$ Furthermore, several large cohort studies reported that weight loss by bariatric surgery reduced considerably all cause- and cause-specific mortality risk. ${ }^{12,13}$ Patients with morbid obesity are likely to take more medications due to multimorbidity associated with obesity. Severe obesity leads to impairment of activities of daily living, which re- sults in distress to patients and aggravation of obesity. ${ }^{1}$ In this regard, it is important to classify obesity to identify the risk of comorbidities and mortality and to establish strategies to address the epidemic of morbid obesity.

The 2020 Obesity Fact Sheet has emphasized a precipitous increasing trend in the prevalence of obesity, particularly in class III obesity, among South Korean young adults. Across the globe, all 
age groups have gained significant weight over the last four decades, with the most rapid weight gain occurring in young adults. Increasing rates of severe obesity in children and adolescents confer a rise in obesity prevalence and premature morbidity and mortality in young adults. Consequently, bariatric surgery has also risen in this population. Severe obesity is associated with emotional and cognitive issues as well as metabolic complications in young adults. ${ }^{14,15}$ Factors associated with obesity may also differ according to age. Young adults tend to have unhealthy dietary habits including frequent consumption of fast food, meal skipping, and eating out, as well as insufficient fruit and vegetable consumption. ${ }^{16} \mathrm{~A}$ study of two cohorts of young adult women born in 1973-1978 and 1989-1995 revealed high weight gain in all sociodemographic groups, but this was most evident in millennial women born in 1989-1995 with high levels of stress and depressive mood. ${ }^{17}$ Meanwhile, a great deal of research has suggested that there is a strongly positive association between obesity and the prevalence of some metabolic complications; however, some literature suggests that the relationship between obesity and metabolic risk may become weaker or stronger with age depending on the risk factor in question. ${ }^{18}$ Therefore, prevention and treatment of obesity in young adults is a global public health priority to decrease the risk of related chronic diseases and mortality in later adulthood.

Health inequalities have been noticed worldwide since the 1980s, and health disparities by sex, socioeconomic status, and region still exist. Moreover, there are still astonishing inter- and intranational differences in health, and regional disparities in the prevalence of chronic diseases including obesity continue to widen. ${ }^{19,20}$ The 2020 Obesity Fact Sheet has reported regional differences in the prevalence of obesity in Korea. There are increasing concerns regarding regional disparities in heathy diet and nutritional status among South Korean adults ${ }^{21}$ and this seems to be one of the factors affecting regional disparities in obesity prevalence in South Korea. One study reported that residents in rural areas had higher odds of an unhealthy diet such as excessive carbohydrates, low fruit, and high salted-vegetable intake compared to those in metropolitan areas. ${ }^{22}$ Differences in lifestyle patterns, health perceptions, environmental and cultural factors, and accessibility to public transportation by region may affect regional disparities in obesity prevalence. Small cities in isolated areas may have difficulty managing obesity due to relatively poor access to medical services and exercise facilities. Further investigation examining modifiable factors related to obesity in each region is needed.

This study has several limitations and strengths. We assessed the prevalence of obesity without considering any factors related to obesity in South Korea. However, this study included nearly the whole adult population of Korea who underwent national health checkups and provides invaluable data on national health statistics with regard to obesity classes. Moreover, we performed stratified analysis by age, sex, and region.

In conclusion, based on the findings of the 2020 Obesity Fact Sheet, the prevalence of class I, II, and III obesity increased between 2009 and 2018, with a dramatic increase of class II and III obesity. The prevalence of obesity overall and all classes increased most dramatically in young adults. Regional differences in obesity prevalence were also found. This study's findings provide a better understanding of the obesity epidemic in South Korea, including variation in prevalence by age and region, and suggest that appropriate strategies for obesity according to obesity class, age, and region are urgently needed.

\section{CONFLICTS OF INTEREST}

Eun-Jung Rhee has worked as an Editor-in-Chief of the journal since 2021. And, Ga Eun Nam has worked as an Associate Editor of the journal since 2020. However, they were not involved in the peer reviewer selection, evaluation, or decision process of this article. Otherwise, no other potential conflicts of interest relevant to this article were reported.

\section{ACKNOWLEDGMENTS}

This work was partially supported by the Korean Society for the Study of Obesity. We cordially thank the National Health Insurance Service and the Korean Society for the Study of Obesity for their support.

\section{AUTHOR CONTRIBUTIONS}

Study concept and design: GEN, YHK, KH, JHJ, EJR, and 
WYL; acquisition of data: GEN, YHK, KH, JHJ, EJR, and WYL; statistical analysis: $\mathrm{KH}$ and JHJ; interpretation of data: GEN, YHK, $\mathrm{KH}, \mathrm{JHJ}, \mathrm{EJR}$, and WYL; drafting of the manuscript: GEN and WYL; critical revision of the manuscript: GEN, YHK, KH, JHJ, EJR, and WYL; and study supervision: WYL.

\section{REFERENCES}

1. Abdelaal M, le Roux CW, Docherty NG. Morbidity and mortality associated with obesity. Ann Transl Med 2017;5:161.

2. Arroyo-Johnson C, Mincey KD. Obesity epidemiology worldwide. Gastroenterol Clin North Am 2016;45:571-9.

3. Nam GE, Kim YH, Han K, Jung JH, Rhee EJ, Lee SS, et al. Obesity fact sheet in Korea, 2019: prevalence of obesity and abdominal obesity from 2009 to 2018 and social factors. J Obes Metab Syndr 2020;29:124-32.

4. Ricci MA, De Vuono S, Scavizzi M, Gentili A, Lupattelli G. Facing morbid obesity: how to approach it. Angiology 2016; 67:391-7.

5. Seo MH, Lee WY, Kim SS, Kang JH, Kang JH, Kim KK, et al. 2018 Korean Society for the Study of Obesity Guideline for the Management of Obesity in Korea. J Obes Metab Syndr 2019;28:40-5.

6. Fouse T, Schauer P. The socioeconomic impact of morbid obesity and factors affecting access to obesity surgery. Surg Clin North Am 2016;96:669-79.

7. Finkelstein EA. How big of a problem is obesity? Surg Obes Relat Dis 2014;10:569-70.

8. Haslam DW, James WP. Obesity. Lancet 2005;366:1197-209.

9. Sarma S, Sockalingam S, Dash S. Obesity as a multisystem disease: trends in obesity rates and obesity-related complications. Diabetes Obes Metab 2021;23 Suppl 1:3-16.

10. Hartemink N, Boshuizen HC, Nagelkerke NJ, Jacobs MA, van Houwelingen HC. Combining risk estimates from observational studies with different exposure cutpoints: a meta-analysis on body mass index and diabetes type 2. Am J Epidemiol 2006; 163:1042-52.

11. Flegal KM, Kit BK, Orpana H, Graubard BI. Association of all-cause mortality with overweight and obesity using standard body mass index categories: a systematic review and meta-analysis. JAMA 2013;309:71-82.

12. Sjöström L, Narbro K, Sjöström CD, Karason K, Larsson B, Wedel $\mathrm{H}$, et al. Effects of bariatric surgery on mortality in Swedish obese subjects. N Engl J Med 2007;357:741-52.

13. Adams TD, Gress RE, Smith SC, Halverson RC, Simper SC, Rosamond WD, et al. Long-term mortality after gastric bypass surgery. N Engl J Med 2007;357:753-61.

14. Horesh A, Tsur AM, Bardugo A, Twig G. Adolescent and childhood obesity and excess morbidity and mortality in young adulthood-a systematic review. Curr Obes Rep 2021 May 5 [Epub]. https://doi.org/10.1007/s13679-021-00439-9

15. Baskaran C, Animashaun A, Rickard F, Toth AT, Eddy KT, Plessow F, et al. Memory and executive function in adolescent and young adult females with moderate to severe obesity before and after weight loss surgery. Obes Surg 2021;31:3372-8.

16. Martin JC, Moran LJ, Harrison CL. Diet quality and its effect on weight gain prevention in young adults: a narrative review. Semin Reprod Med 2020;38:407-13.

17. Brown WJ, Flores TR, Keating SE, Mielke GI. Trajectories and determinants of weight gain in two cohorts of young adult women born 16 years apart. Int J Obes (Lond) 2021; 45:1553-64.

18. Canning KL, Brown RE, Jamnik VK, Kuk JL. Relationship between obesity and obesity-related morbidities weakens with aging. J Gerontol A Biol Sci Med Sci 2014;69:87-92.

19. Ng M, Fleming T, Robinson M, Thomson B, Graetz N, Margono C, et al. Global, regional, and national prevalence of overweight and obesity in children and adults during 19802013: a systematic analysis for the Global Burden of Disease Study 2013. Lancet 2014;384:766-81.

20. Lee KW, Shin D. Relationships of dietary factors with obesity, hypertension, and diabetes by regional type among single-person households in Korea. Nutrients 2021;13:1218.

21. Lee J, Sa J. Regional disparities in healthy eating and nutritional status in South Korea: Korea National Health and Nutrition Examination Survey 2017. Nutr Res Pract 2020;14:679-90.

22. Ha K, Song Y, Kim HK. Regional disparities in the associations of cardiometabolic risk factors and healthy dietary factors in Korean adults. Nutr Res Pract 2020;14:519-31. 